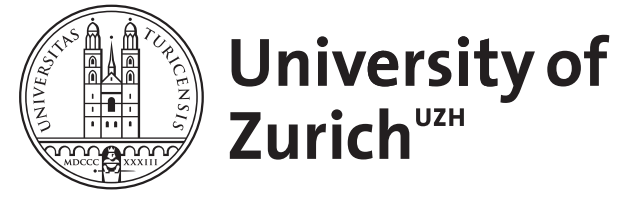

\title{
Gamma-oscillations from bench to bed
}

Grunwald, T ; Sarnthein, J

\begin{abstract}
In the presurgical evaluation of patients with pharmacoresistant epilepsies, the borders between disciplines tend to oscillate. In particular, the necessity to evaluate the functional capacity of a cortical area that has been shown to be epileptogenic and is thus being considered for resection often makes it impossible not to trespass between neurophysiology and neuropsychology. In epilepsy surgery, the principle of "first, do no harm", often blurs the distinction between "bed and bench": findings from basic sciences that implicate a certain brain region in specific neurological or neuropsychological functions must be taken into account when an elective resection of a structure is considered - even if resection might relieve the patient from frequent intolerable seizures. That may seem to go without saying and, in fact, several examinations that are used routinely in modern presurgical evaluation are really shortcuts in the path from bench to bed. It may be an excuse that there are still are no generally accepted protocols for examinations such as the intracarotid amobarbital ("Wada") test or language mapping by electrostimulation, be the latter intrasurgical or by chronically implanted subdural electrodes. On the other hand, the opposite way "from bed to bench and back" has a famous history in epilepsy surgery: a new area of memory research followed Brenda Milner's analysis of the famous anterograde amnesia caused by the resection of both hippocampi in Henry Molaison (H.M.) and, eventually, this has led to important clinical applications in both neuropsychology and neurophysiology.
\end{abstract}

DOI: https://doi.org/10.1016/j.clinph.2012.02.083

Posted at the Zurich Open Repository and Archive, University of Zurich

ZORA URL: https://doi.org/10.5167/uzh-72863

Journal Article

Accepted Version

Originally published at:

Grunwald, T; Sarnthein, J (2012). Gamma-oscillations from bench to bed. Clinical Neurophysiology, 123(10):1897-1898.

DOI: https://doi.org/10.1016/j.clinph.2012.02.083 


\section{Manuscript}

Grunwald T, Sarnthein J.

\section{Gamma-oscillations from bench to bed}

In the presurgical evaluation of patients with pharmacoresistant epilepsies, the borders between disciplines tend to oscillate. In particular, the necessity to evaluate the functional capacity of a cortical area that has been shown to be epileptogenic and is thus being considered for resection often makes it impossible not to trespass between neurophysiology and neuropsychology. In epilepsy surgery, the principle of "first, do no harm", often blurs the distinction between "bed and bench": findings from basic sciences that implicate a certain brain region in specific neurological or neuropsychological functions must be taken into account when an elective resection of a structure is considered - even if resection might relieve the patient from frequent intolerable seizures. That may seem to go without saying and, in fact, several examinations that are used routinely in modern presurgical evaluation are really shortcuts in the path from bench to bed. It may be an excuse that there are still are no generally accepted protocols for examinations such as the intracarotid amobarbital ("Wada") test or language mapping by electro-stimulation, be the latter intrasurgical or by chronically implanted subdural electrodes. On the other hand, the opposite way "from bed to bench and back" has a famous history in epilepsy surgery: a new area of memory research followed Brenda Milner's analysis of the famous anterograde amnesia caused by the resection of both hippocampi in Henry Molaison (H.M.) and, eventually, this has led to important clinical applications in both neuropsychology and neurophysiology.

By contrast, gamma-oscillations were initially implicated in the binding of neuronal representations of different but associated attributes of visual stimuli by "bench-sided" laboratory studies (Gray et al., 1989; for review see Buzsáki, 2006). Gamma oscillations indicative of binding processes have also been identified in the human brain - both in large-scale (e.g. Ribary et al., 1991) and in more regionalized cortical systems, associated with visual (Tallon-Baudry et al., 1997; von Stein and Sarnthein, 2000), lexical (Lutzenberger et al., 1994) and memory processes (Miltner et al., 1999). Even more localized patterns of gamma activity have been found in invasive recordings from subdural and depth electrodes implanted in patients with pharmacoresistant epilepsies undergoing invasive presurgical evaluations for possible epilepsy surgery (e.g. Sederberg et al., 2003; Fell and Axmacher, 2011). These and numerous other 
studies suggest that gamma-oscillations are not confined to specific perceptual modalities or cognitive processes of the brain but can be associated with various functions depending on the brain area(s) in which they occur.

Since invasive recordings during the presurgical evaluation of epilepsy patients offer a unique opportunity to record gamma activity directly from the human brain, it was logical to ask whether modulations of gamma activity can also be used to map eloquent areas of the cortex. Normally these regions must be spared during epilepsy surgery, especially when they subserve motor or language functions. In patients with chronically implanted subdural grid electrodes, cortical areas subserving language processes are normally delineated by mapping those areas where electrical stimulation can interfere with the production and/or perception of spoken or written language. Several earlier studies have shown that gamma activity in the same or overlapping cortical areas can be modulated by language tasks such as listening to or repeating words (e.g. Crone et al., 2001a; Crone et al., 2001b; Towle et al., 2008; Wu et al., 2011).

In this issue, Kojima et al. (2012) take gamma-oscillations a step further to the bedside by comparing language mapping via electrical brain stimulation to mapping using event-related gamma-oscillations. Using a task in which patients were asked simple questions (e.g. "What do you hear with?") and gave short answers ("Ears"), the authors analyzed augmentation of gamma-activity during question and answer in language-related sites in the superior temporal and anterior frontal gyri. Comparing sites that were mapped to be language-related by both methods, they found a higher sensitivity for the language task of gamma-oscillations than electrical stimulation. A true comparison of the specificities of the two methods is out of reach, since this would require comparison of the language deficits after neurosurgical resection of sites indicated to be language-related by the two methods. This is impossible because electrical stimulation is the gold standard of functional mapping, and cortical areas shown to be eloquent for language functions by this method cannot be resected. However, since Kojima et al. found gamma-augmentation in areas that were surgically removed without causing dysphasic symptoms, it seems that cortical electro-stimulation has a higher specificity than the mapping of gamma-augmentation. 
On the other hand, electro-stimulation mapping can also have false negative results (e.g. Kral et al., 2006). Also Kojima et al. (this issue) observed dysphasic symptoms in one patient in whom the removed cortical sites were presumably language-related with event-related augmentation of gamma-activity but not with electrostimulation. Thus, the findings of the present study will make it difficult to negect gamma-oscillations at the bed-side in the future, especially in patients in whom electro-stimulation elicits after-discharges, or even seizures that prevent functional mapping.

Much work still remains to be done at the bench-side to increase the sensitivity and specificity of gamma-oscillation studies for functional mapping of eloquent cortex. It seems likely that gammaoscillations may also subserve different cognitive processes depending on when and exactly where they can be elicited. The same holds true for gamma activity in language tasks that tap into not only speech production and perception but also short-and long-term memory processes. In sum, the paper by Kojima et al. published in the present issue will definitely prove to be important for the presurgical evaluation of epilepsy patients, and it will certainly help to make event-related gamma activity further oscillate between bed and bench and back.

Thomas Grunwald

Schweizerisches Epilepsie-Zentrum

Bleulerstrasse 60

CH 8008 Zürich, Switzerland

$\mathrm{Tel}+41443876111$

Thomas.Grunwald@swissepi.ch

Johannes Sarnthein

Klinik für Neurochirurgie

UniversitätsSpital Zürich

Frauenklinikstrasse 10

CH 8091 Zürich, Switzerland 


\section{References}

Buzsáki, G, 2006. Rhythms of the Brain. Oxford University Press, New York.

Crone, NE, Boatman, D, Gordon, B, Hao, L. Induced electrocorticographic gamma activity during auditory perception. Brazier Award-winning article, 2001. Clin Neurophysiol 2001a; 112: 565582.

Crone, NE, Hao, L, Hart, J, Jr., Boatman, D, Lesser, RP, Irizarry, R, et al. Electrocorticographic gamma activity during word production in spoken and sign language. Neurology 2001b; 57: 2045-2053.

Fell, J, Axmacher, N. The role of phase synchronization in memory processes. Nat Rev Neurosci 2011; 12: 105-118.

Gray, CM, König, P, Engel, AK, Singer, W. Oscillatory responses in cat visual cortex exhibit intercolumnar synchronization which reflects global stimulus properties. Nature 1989; 338: 334-337.

Kojima K, Brown EC, Rothermel R, Carlson A, Matsuzaki N, Shah A, Atkinson M, Mittal S, Fuerst D, Sood S, Asano E. Multimodality language mapping in patients with left-hemispheric language dominance on Wada test. Clin Neurophysiol 2012; xx: xxx-xxx.

Kral, T, Kurthen, M, Schramm, J, Urbach, H, Meyer, B. Stimulation mapping via implanted grid electrodes prior to surgery for gliomas in highly eloquent cortex. Neurosurgery 2006; 58: ONS3643; discussion ONS36-43.

Lutzenberger, W, Pulvermüller, F, Birbaumer, N. Words and pseudowords elicit distinct patterns of 30 $\mathrm{Hz}$ EEG responses in humans. Neurosci Lett 1994; 176: 115-118.

Miltner, WH, Braun, C, Arnold, M, Witte, H, Taub, E. Coherence of gamma-band EEG activity as a basis for associative learning. Nature 1999; 397: 434-436.

Ribary, U, loannides, AA, Singh, KD, Hasson, R, Bolton, JP, Lado, F, et al. Magnetic field tomography of coherent thalamocortical $40-\mathrm{Hz}$ oscillations in humans. Proc Natl Acad Sci U S A 1991; 88: 11037-11041.

Sederberg, PB, Kahana, MJ, Howard, MW, Donner, EJ, Madsen, JR. Theta and gamma oscillations during encoding predict subsequent recall. J Neurosci 2003; 23: 10809-10814.

Tallon-Baudry, C, Bertrand, O, Delpuech, C, Permier, J. Oscillatory gamma-band $(30-70 \mathrm{~Hz})$ activity induced by a visual search task in humans. J Neurosci 1997; 17: 722-734.

Towle, VL, Yoon, HA, Castelle, M, Edgar, JC, Biassou, NM, Frim, DM, et al. ECoG gamma activity during a language task: differentiating expressive and receptive speech areas. Brain 2008; 131 : 2013-2027.

von Stein, A, Sarnthein, J. Different frequencies for different scales of cortical integration: from local gamma to long range alpha/theta synchronization. Int J Psychophysiol 2000; 38: 301-313.

Wu, HC, Nagasawa, T, Brown, EC, Juhasz, C, Rothermel, R, Hoechstetter, K, et al. gammaoscillations modulated by picture naming and word reading: intracranial recording in epileptic patients. Clin Neurophysiol 2011; 122: 1929-1942. 